\title{
Membrane glycoprotein M6A promotes $\mu$-opioid receptor endocytosis and facilitates receptor sorting into the recycling pathway
}

\author{
Ying-Jian Liang ${ }^{1}$, Dai-Fei Wu ${ }^{1}$, Ralf Stumm ${ }^{1}$, Volker Höllt ${ }^{1}$, Thomas Koch ${ }^{1}$ \\ ${ }^{1}$ Department of Pharmacology and Toxicology, Otto-von-Guericke University, Leipziger Str. 44, 39120 Magdeburg, Germany
}

\begin{abstract}
The interaction of $\mu$-opioid receptor (MOPr) with the neuronal membrane glycoprotein M6a is known to facilitate MOPr endocytosis in human embryonic kidney 293 (HEK293) cells. To further study the role of M6a in the post-endocytotic sorting of MOPr, we investigated the agonist-induced co-internalization of MOPr and M6a and protein targeting after internalization in HEK293 cells that co-expressed HA-tagged MOPr and Myc-tagged M6a. We found that M6a, MOPr, and Rab 11, a marker for recycling endosomes, co-localized in endocytotic vesicles, indicating that MOPr and M6a are primarily targeted to recycling endosomes after endocytosis. Furthermore, co-expression of M6a augmented the post-endocytotic sorting of $\delta$-opioid receptors into the recycling pathway, indicating that M6a might have a more general role in opioid receptor post-endocytotic sorting. The enhanced post-endocytotic sorting of MOPr into the recycling pathway was accompanied by a decrease in agonist-induced receptor down-regulation of M6a in co-expressing cells. We tested the physiological relevance of these findings in primary cultures of cortical neurons and found that co-expression of M6a markedly increased the translocation of MOPrs from the plasma membrane to intracellular vesicles at steady state and significantly enhanced both constitutive and agonist-induced receptor endocytosis. In conclusion, our results strongly indicate that M6a modulates MOPr endocytosis and post-endocytotic sorting and has an important role in receptor regulation.
\end{abstract}

Keywords: $\mu$-opioid receptor, membrane glycoprotein M6a, internalization, recycling, post-endocytotic sorting

Cell Research (2008) 18:768-779. doi: 10.1038/sj.cr.2008.71; published online 24 June 2008

\section{Introduction}

Opiates like morphine remain the best analgesic for treating chronic and serious pain. After prolonged or repeated drug administration, however, adaptive changes in the nervous system occur that are believed to contribute to physiological tolerance of and dependence on opiates and thereby limit the clinical utility of these drugs. These adaptive changes are initiated by the binding of opiate drugs to opioid receptors, which are also activated by endogenous opioid neuropeptides [1]. There are three well-defined 'classical' types of the opioid receptors, $\mu$-, $\delta$ - and $\kappa$-opioid receptors, which belong to the superfamily of G protein-coupled receptors (GPCRs) [2]. The $\mu$-opioid receptor (MOPr) mediates the action of most clinically

Correspondence: Thomas Koch

Tel: +49-391-6715372; Fax: +49-391-6715869

E-mail: thomas.koch@Medizin.Uni-Magdeburg.de

Received 28 August 2007; revised 22 January 2008; accepted 26 February 2008; published online 24 June 2008 important opiate drugs, like morphine, as well as drugs of abuse, such as heroin [3-5]. Understanding the molecular mechanisms underlying the regulation of opioid receptors is a key step in the development of drugs and/or therapies that provide effective analgesia without causing detrimental adaptive responses.

As with other GPCRs, receptor phosphorylation occurs upon agonist binding. $\beta$-Arrestins then bind to phosphorylated receptors, causing the receptor to uncouple from G-proteins and leading to the targeting of receptors to clathrin-coated pits. Once the plasma membrane invaginates, vesiculation occurs as the GTPase dynamin wraps around the neck of the invagination and constricts upon GTP hydrolysis. These vesicles soon shed their clathrin coats and become early endosomes. Dissociation of ligands and $\beta$-arrestins from the receptors occurs in an acidified perinuclear compartment. Cytosolic phosphatases may dephosphorylate the receptors. The ligands are degraded while the receptors are either recycled to the plasma membrane for reactivation or downregulated by means 
of degradation in lysosomes [6-11]. Receptor-associated proteins play important roles during receptor endocytosis and intracellular trafficking. A number of proteins that bind to the MOPr have been recently identified and functionally characterized [12-16]. We have previously identified and characterized a novel MOPr-interacting protein, the membrane glycoprotein M6a, which interacts with the MOPr and plays a role in receptor endocytosis in transfected human embryonic kidney 293 (HEK293) cells [17]. Here, we demonstrate that the co-expression of M6a and MOPr in HEK293 cells facilitates the post-endocytotic sorting of internalized opioid receptors into the recycling pathway and decreases receptor proteolytic down-regulation. Importantly, we also present evidence from work in primary cultured neurons to suggest that M6a has a similar function in native neurons.

\section{Results}

\section{Membrane glycoprotein M6a is a trafficking molecule}

We recently reported that M6a facilitates the agonistinduced and constitutive endocytosis of the MOPr in transfected HEK293 cells [17]. To investigate the trafficking of M6a in the absence of MOPr, we stably expressed an amino-terminally Myc-tagged M6a in HEK293 cells. As depicted in Figure 1, M6a localizes to both the plasma membrane and cytosolic vesicles in stably transfected HEK293 cells. Interestingly, after exposure to monensin, an inhibitor of vesicle recycling, M6a accumulates in cytosolic vesicle-like compartments. In contrast, after exposure to hypertonic sucrose (which blocks protein internalization via clathrin-coated pits), M6a is significantly fixed at the plasma membrane. These data confirm that M6a undergoes constitutive endocytosis and recycling.

M6a facilitates the recycling pathway of opioid receptors

To determine whether M6a modulates the post-endocytotic sorting of opioid receptors, we stably expressed an $\mathrm{N}$-terminally HA-tagged MOPr or DOPr together with a Myc-tagged M6a in HEK293 cells. Numerous publications have shown that the MOPr and $\delta$-opioid receptor differ in their post-endocytotic sorting [16, 18-20]. In fact, internalized DOPr is known to be mainly sorted to the lysosome and degraded, whereas internalized MOPr is mainly sorted to the recycling endosomes for reactivation. Since M6a exhibits a strong interaction with $M O P r$ [17], we carried out a 'pulse-chase' experiment to examine whether internalized M6a, together with MOPr, could be sorted to the recycling endosome. As expected, in HEK293 cells coexpressing MOPr and M6a, we found a remarkable co-localization of M6a and MOPr with transferrin, a marker for recycling endosomes [21, 22], in endocytotic vesicles (Figure 2A, arrow). In DOPr-expressing cells, no co-localization of DOPr with transferrin could be detected in endocytotic vesicles (Figure 2B, arrow head). Since M6a has also been shown to interact with DOPr [17], we investigated whether the interaction of M6a with DOPr could recruit internalized DOPr into recycling vesicles. As shown in Figure 2B (arrow), upon co-expression with M6a, internalized DOPr could be found to colocalize with transferrin in endocytotic vesicles. These data suggest that M6a might direct internalized opioid receptors into recycling endosomes during post-endocytotic sorting.

Since transferrin labels recycling endosomes as well

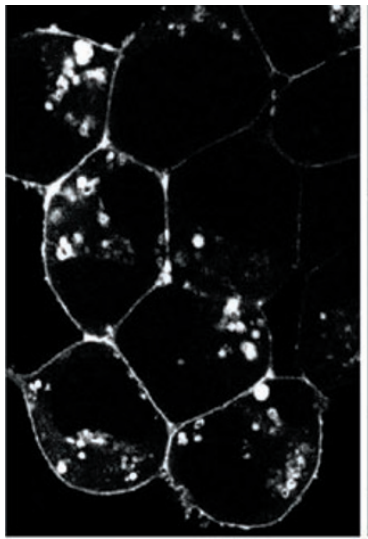

Control

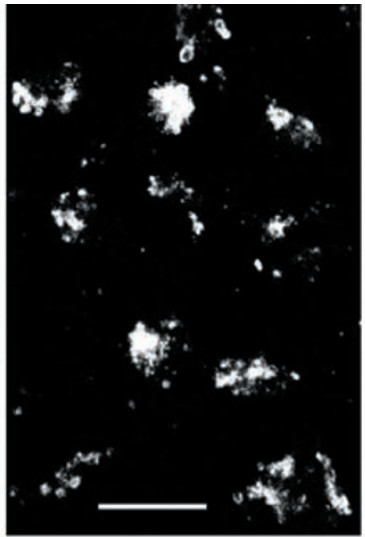

Monensin

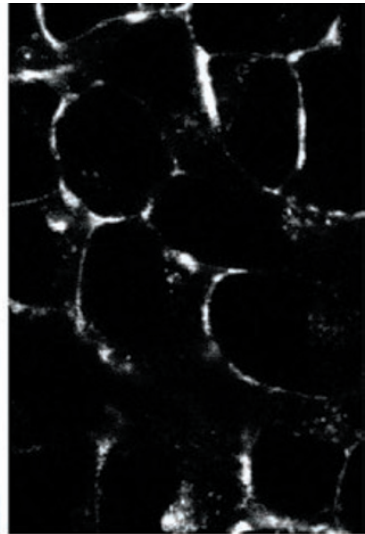

Sucrose

Figure 1 Intracellular trafficking of the membrane glycoprotein M6a. HEK293 cells stably expressing N-terminally Myc-tagged M6a were either left unexposed (control) or exposed to $50 \mu \mathrm{M}$ monensin or $400 \mathrm{mM}$ sucrose at $37^{\circ} \mathrm{C}$ for $2 \mathrm{~h}$. The cells were subsequently fixed, and the distribution of M6a was examined by confocal microscopy as described in 'Materials and Methods'. Representative images from two independent experiments are shown. Scale bar, $16 \mu \mathrm{m}$. 


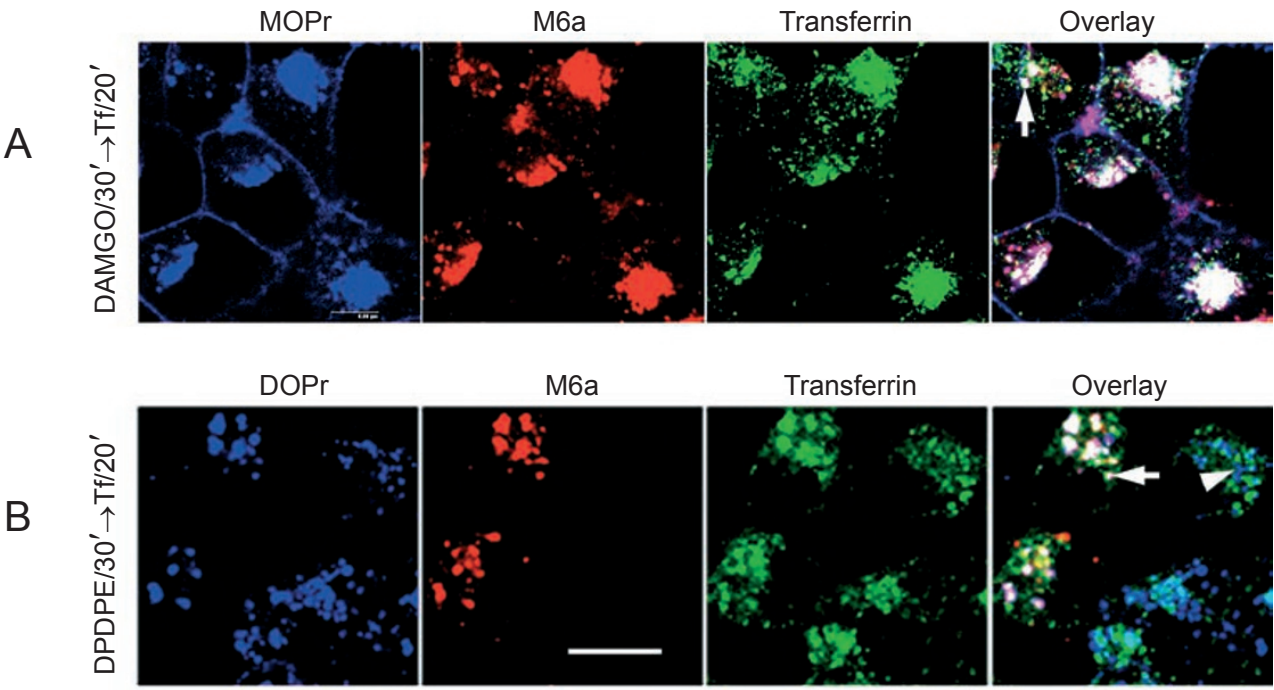

Figure 2 Pulse-chase analysis of internalized MOPr and M6a relative to endocytosed transferrin. Surface opioid receptors (MOPr, DOPr) and M6a (A, B) were labeled by exposing intact cells to appropriate specific antibodies. To analyze the post-endocytotic sorting of internalized receptors and $\mathrm{M} 6 \mathrm{a}$, confocal microscopy of triple-labeled cells was used to examine the co-localization of a 30-min agonist pulse of antibody-labeled receptors and M6a, followed by a 20-min chase with transferrin in the absence of agonist, as described under 'Materials and Methods'. Note that the majority of vesicles containing both internalized MOPr/DOPr and M6a also contain transferrin (arrow); however, many vesicles containing internalized DOPr alone do not contain transferrin (arrow head), while vesicles containing MOPr alone do contain transferrin (data not shown). overlay of MOPr (blue), M6a (red) and transferrin (green) is denoted by the white vesicular structures visualized in the merged image. Overlay of receptor with M6a generated magenta; Overlay of receptor with transferrin generated cyan; and overlay of M6a with transferrin generated yellow. Representative images from one of three independent experiments are shown. Scale bar, $16 \mu \mathrm{m}$.

as early endosomes, we next investigated whether M6a could influence the distribution of internalized receptors in specific endosomes. We found that acute agonist treatment (15 min) induced the co-internalization of MOPr-M6a or DOPr-M6a and their localization to EEA1-labeled early endosomes (Figure 3A, upper and lower panels, respectively). Internalized receptors, with or without co-expression of M6a, primarily localized to EEA1-labeled early endosomes (Figure 3A). Longer agonist treatments reduced the localization to early endosomes (data not shown). When the cells were stimulated for $30 \mathrm{~min}$ with MOPr agonist DAMGO or DOPr agonist DPDPE and then incubated for $15 \mathrm{~min}$ in agonist-free medium, there was a significant difference in the distribution of internalized receptors in Rab 11-labeled recycling endosomes. For internalized MOPr, agonist withdrawal led to receptor localization in Rab 11-labeled recycling endosomes; co-expression of M6a with MOPr increased this localization (Figure 3B, upper panel). In contrast, for internalized DOPr, after agonist withdrawal the receptor localization to Rab 11-labeled recycling endosomes was weak, although co-expression of M6a with DOPr also enhanced endosomal localization of DOPr (Figure 3B, lower panel). As depicted in Figure 3B, co-internalized DOPr-M6a was localized to Rab 11- labeled recycling endosomes (arrow, lower panel), whereas adjacent vesicles containing only internalized DOPr were not labeled by the anti-Rab 11 antibody (arrow head, lower panel), indicating that M6a directs internalized DOPr into recycling endosomes during post-endocytotic sorting. Furthermore, we quantified the percent localization of internalized receptors in EEA1-labeled early endosome or Rab 11-labeled recycling endosome. As depicted in Figure 3C, M6a did not significantly influence the localization of internalized receptors in the early endosome after acute agonist treatment; however, co-internalization of M6a significantly increased the localization of internalized receptors at the recycling endosome, especially for internalized DOPr. This result demonstrates that co-internalization of M6a with opioid receptors can influence the receptor recycling pathway in post-endocytotic sorting.

M6a attenuates agonist-induced down-regulation of the $\mathrm{MOPr}$

Down-regulation of opioid receptors in response to chronic opioid treatment is a long-term adaptive process that results from the proteolytic degradation of internalized receptors. Our data suggest that co-expression of M6a can facilitate the postendocytotic sorting of opioid 
A
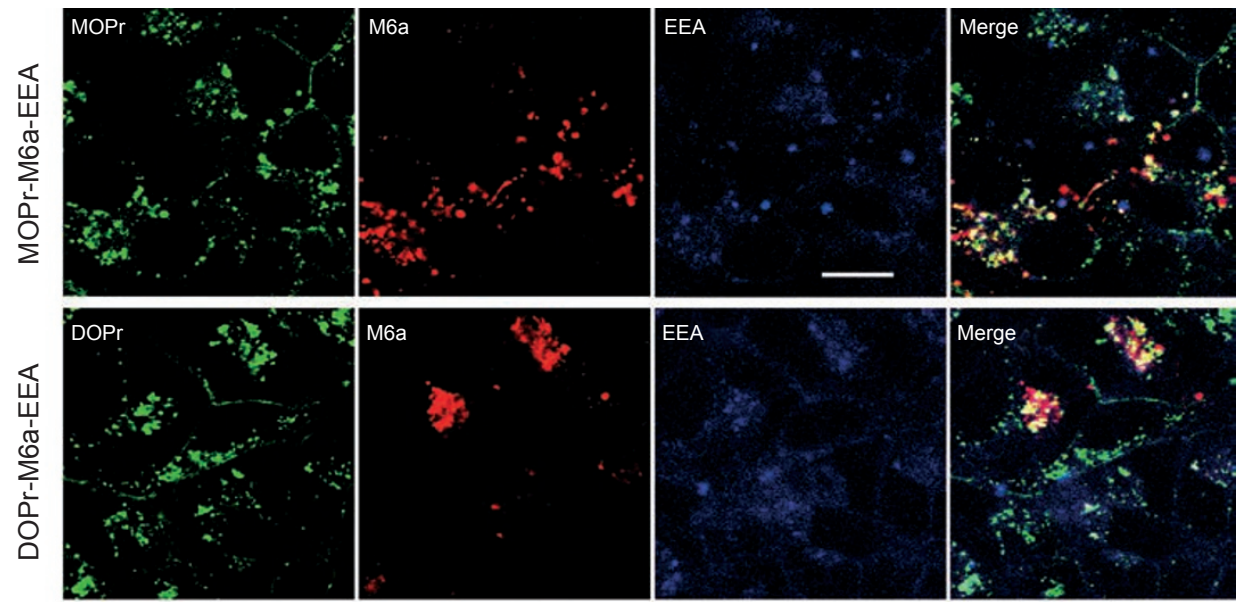

B
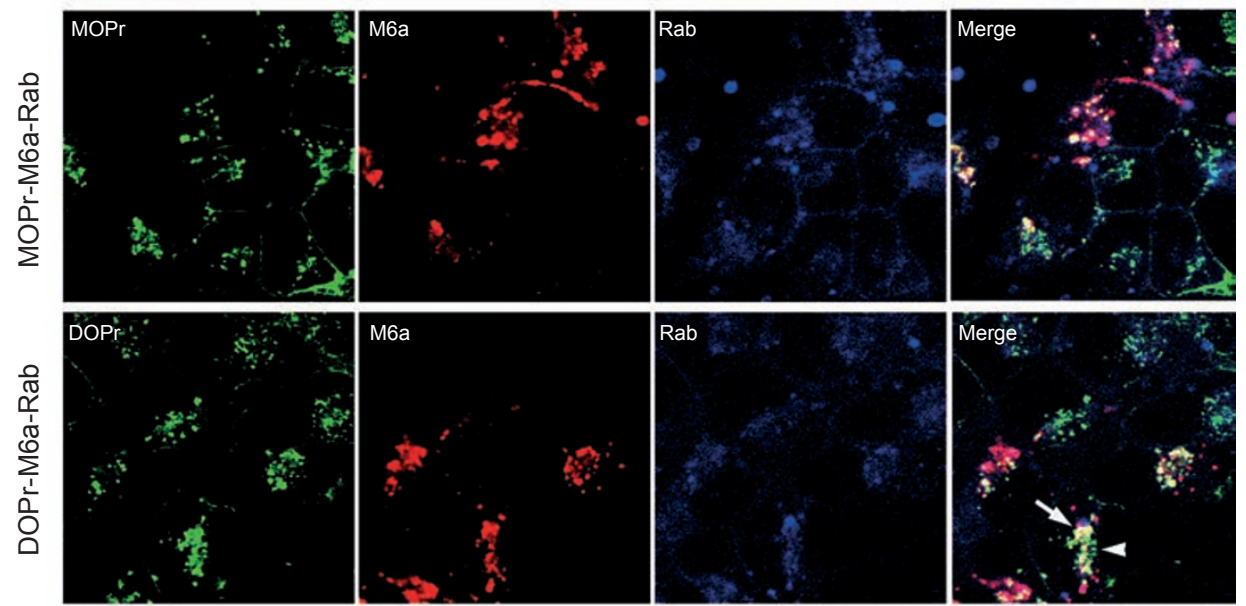

C

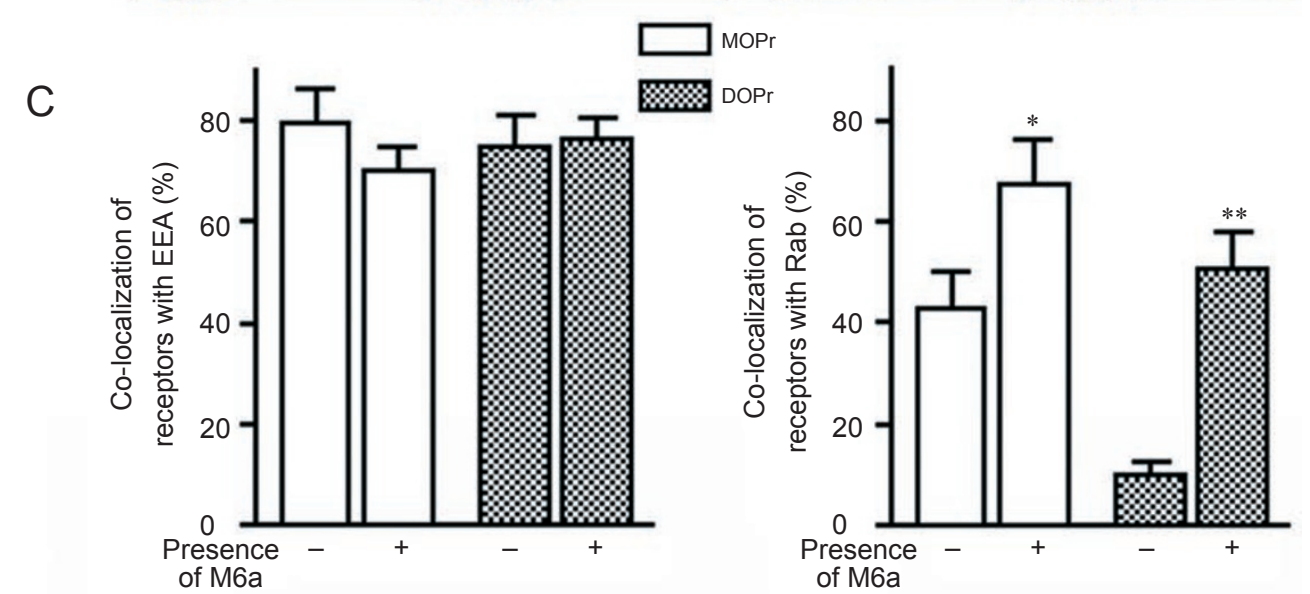

MOPr

Figure $3 \mathrm{M} 6 \mathrm{a}$ increases accumulation of opioid receptors in recycling endosomes. Surface opioid receptors (MOPr and DOPr) and M6a were labeled by exposing intact cells to appropriate specific antibodies. (A) To characterize the endocytic vesicles of internalized receptors and M6a, the cells were stimulated with $10 \mu \mathrm{M}$ DAMGO (upper panel) or $1 \mu \mathrm{M}$ DPDPE (lower panel) for $15 \mathrm{~min}$. (B) To characterize the recycling of internalized receptors and M6a, the cells were firstly stimulated with $10 \mu \mathrm{M}$ DAMGO (upper panel) or $1 \mu \mathrm{M}$ DPDPE (lower panel) for $30 \mathrm{~min}$. Subsequently, the agonists were washed, and the cells were incubated in agonist-free medium for an additional $15 \mathrm{~min}$. After treatment (A, B), the cells were fixed and permeabilized. The distribution of receptors, M6a, EEA1 and/or Rab 11 is described in 'Materials and Methods'. Overlay of MOPr (green), M6a (red) and EEA1/Rab 11, (blue) is shown as white vesicular structures in the merged image. Representative images from one of three independent experiments are shown. Scale bar, $16 \mu \mathrm{m}$. (C) Quantitative analysis of the co-localization of receptors with early endosome marker EEA1 and recycling endosome marker Rab 11. As described in (A, B), the vesicles with co-localization of receptors, M6a and/or EEA1/Rab 11 were counted. Data are presented as means \pm SEM from about 100 cells in two independent experiments. ${ }^{*} P<0.05,{ }^{*} P<0.001$ versus in the absence of M6a, respectively (Student's $t$-test). 
receptors into the recycling pathway. It is reasonable to suggest, therefore, that M6a-enhanced MOPr recycling might decrease receptor endosomal sorting to lysosomes for degradation and thus decrease receptor down-regulation. In fact, we found that agonist-induced down-regulation of MOPr was significantly reduced in cells that were co-expressing M6a (Figure 4). After an extended exposure (16 h) to DAMGO, we detected that only about $25 \%$ of MOPr were downregulated in cells that were co-expressing $\mathrm{MOPr}$ and M6a, compared to about $42 \%$ down-regulation in cells expressing MOPr alone (Figure 4).

\section{M6a increases the intracellular localization of MOPr in cortical neurons}

We next tested whether M6a affects MOPr regulation in primary cultured neurons of the rat cortex. Since endogenous expression of MOPr and M6a proteins in cultured cortical neurons from the embryonic day 17 embryos was below the detection level for immunocytochemistry and/or a functional assay (data not shown), we transiently transfected the neurons with vectors that drive expression of MOPr and M6a. The expression of MOPr and/or M6a proteins could be detected between 1 day and 2 weeks after transfection. Using trypan blue staining to distinguish between dead and live cells, we found that exogenous expression of MOPr and/or M6a did not influence the health of the neurons during the 2 weeks of culture (data not shown).

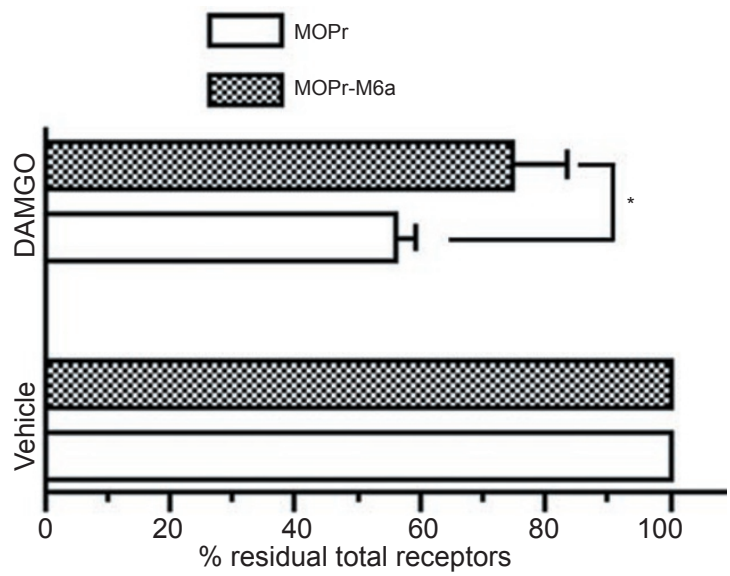

Figure 4 Effect of M6a on agonist-induced down-regulation of $\mu$ opioid receptors. HEK293 cells stably expressing HA-MOPr with and without co-expression of Myc-M6a were incubated at $37^{\circ} \mathrm{C}$ for $16 \mathrm{~h}$ in the presence or absence of $5 \mu \mathrm{M}$ DAMGO. The total amount of MOPr was examined by a membrane binding assay, as described in 'Materials and Methods'. The binding of total receptors without DAMGO treatment was set as $100 \%$ (Vehicle). Values represent mean \pm SEM of 3-5 independent measurements performed in triplicate. ${ }^{*} P<0.05$ : indicates significant difference between MOPr and MOPr-M6a expressing cells (Student's $t$-test).
At 5-6 days after transfection, the neurons were viable and well-developed and thus were used in the following experiments. Werner et al. [23] reported that endogenous M6A localizes to the cell surface and is much less abundant in intracellular compartments. Endogenous MOPr is mainly localized to the plasma membrane of neurons [24]. In our cultured cortical neurons expressing either M6a or MOPr, M6a was predominantly localized to intracellular vesicles (Figure 5A), whereas MOPr was predominantly localized to the plasma membrane (Figure 5B). Similar to our previous findings in HEK293 cells [17], the co-expression of MOPr with M6a significantly increased the intracellular localization of MOPrs (Figure 5C) compared with cultured cortical neurons expressing MOPr alone (Figure 5B). These findings suggest that M6a can enhance the constitutive endocytosis of MOPr in neurons.

Co-expression of M6a augments agonist-induced and constitutive MOPr endocytosis in cortical neurons

Next, we performed surface-labeling to investigate the constitutive and agonist-induced endocytosis of membrane MOPr and M6a in cortical neurons. As shown in Figure 6A and 6B, M6a and MOPr were specifically surface-labeled at the plasma membrane of both the cell body and processes. After 30 min at $37^{\circ} \mathrm{C}$ without agonist treatment, constitutive receptor endocytosis was hardly visible in neurons expressing MOPr alone (Figure 6C), whereas co-expression of M6a with MOPr increased constitutive co-internalization of MOPr and M6a (Figure 6D, yellow vesicles). In addition, agonist-induced MOPr endocytosis after $30 \mathrm{~min}$ of DAMGO treatment (Figure 6E) was enhanced by the co-expression of M6a (Figure 6F). These findings indicate that MOPr and M6a are colocalized and co-internalized in primary cortical neurons and that M6a enhances the constitutive and agonist-induced MOPr internalization.

We further quantified the effect of M6a on MOPr endocytosis. As depicted in Figure 7A, co-expression of M6a significantly enhanced both constitutive and agonistinduced endocytosis of MOPrs. Although M6a and DM20 share high homology and belong to the same proteolipid protein (PLP) family of tetraspan membrane proteins [25], co-expression of DM20 did not influence MOPr endocytosis. A shorter truncation mutant (129-255 aa) of M6a was previously shown to interact with MOPr and attenuate agonist-induced receptor endocytosis [17]. Consistent with that finding in transfected HEK293 cells [17], co-expression of this dominant negative M6a truncation mutant (nM6a) significantly decreased MOPr endocytosis (Figure 7A). Moreover, through imaging using a confocal microscope, we found that the $\mu$-agonist DAMGO clearly induced co-internalization and co-localization of MOPr and M6a (Figure 6D and 6F), whereas it failed to cause co-localiza- 
A
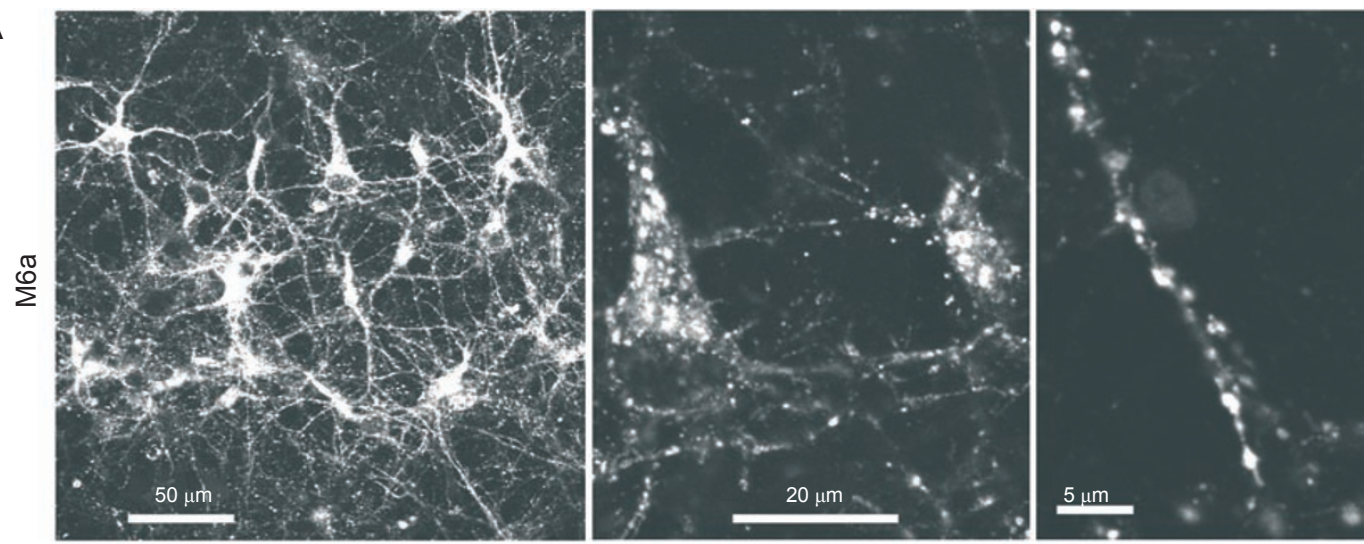

B
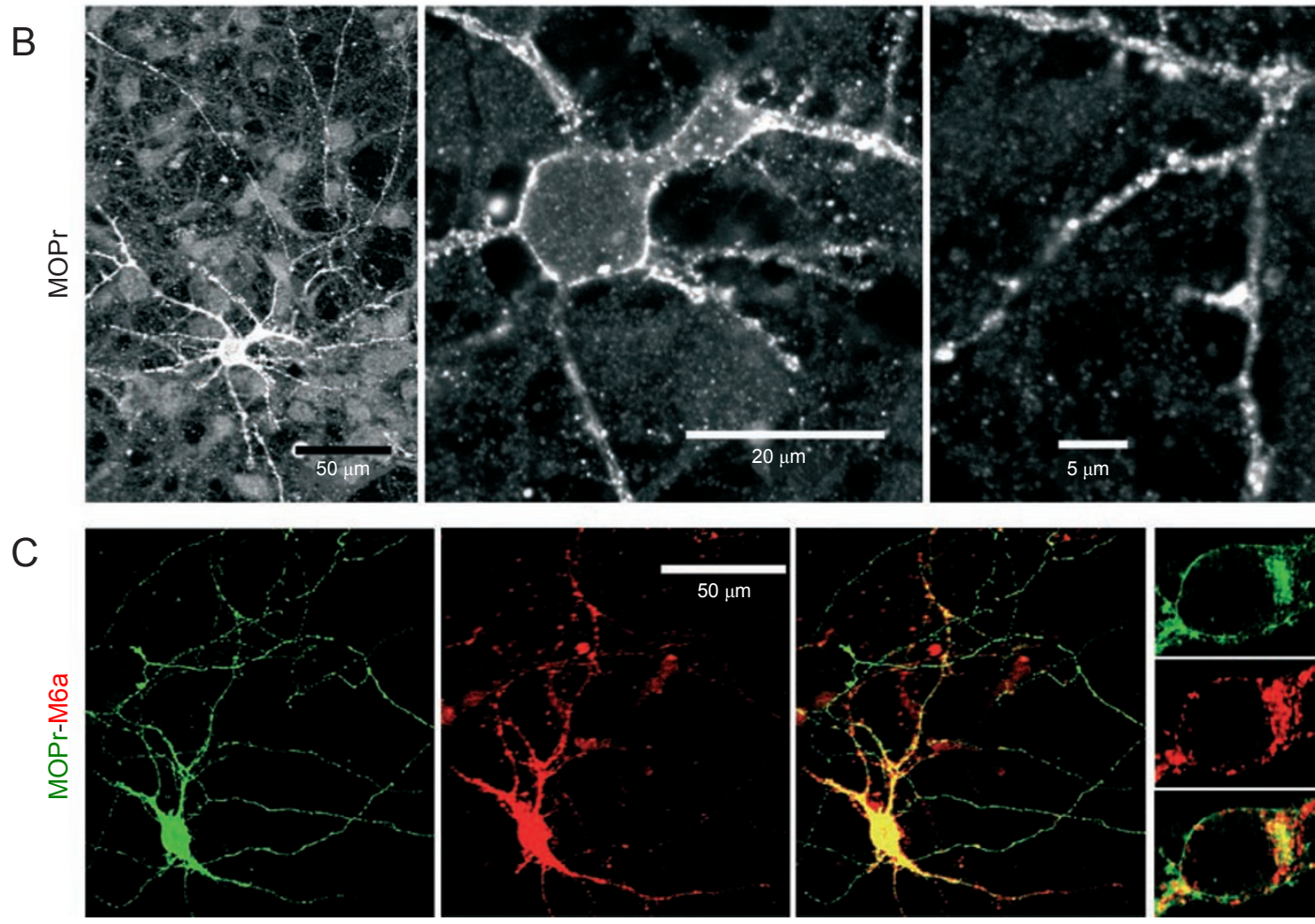

Figure $5 \mathrm{M} 6 \mathrm{a}$ increases the intracellular localization of MOPr in transfected cortical neurons. Newly prepared cortical neurons (E17) were transfected with Myc-M6a (A), HA-MOPr (B) or Myc-M6a + HA-MOPr (C) plasmids using a 'Rat Neuron Nucleofector Solution' and then seeded in a poly-L-lysine-coated plate. At 6 days after transfection, the cells were fixed. HA-MOPr and Myc-M6a were detected using anti-HA and/or anti-Myc antibodies as described in 'Materials and Methods'. The cell body and/or process are shown with a higher magnification. Note that co-expression of M6a significantly increases the intracellular localization of MOPrs. Overlay of MOPr (green) and M6a (red) is shown as a yellow color visualized in the merged image. Shown is a representative result from one of three independent experiments. Scale bars are shown as indicated.

tion of MOPr and DM20 (Figure 7B). In addition, in many neurons, the co-expression of nM6a markedly decreased agonist-induced MOPr endocytosis (Figure 7C). These findings indicate that M6a-enhanced MOPr endocytosis in neurons is due to M6a itself.

\section{Discussion}

The membrane glycoprotein M6a is a member of the PLP family of tetraspan membrane proteins and is expressed primarily in neurons [25-27]. It is generally believed that glycoproteins of the PLP family function as structural proteins for myelination. M6a, however, is also thought to function as a modulator for neurite outgrowth [28] and spine formation [29], and also acts as a nerve growth factor-gated $\mathrm{Ca}^{2+}$ channel in neuronal differentiation [30]. The 

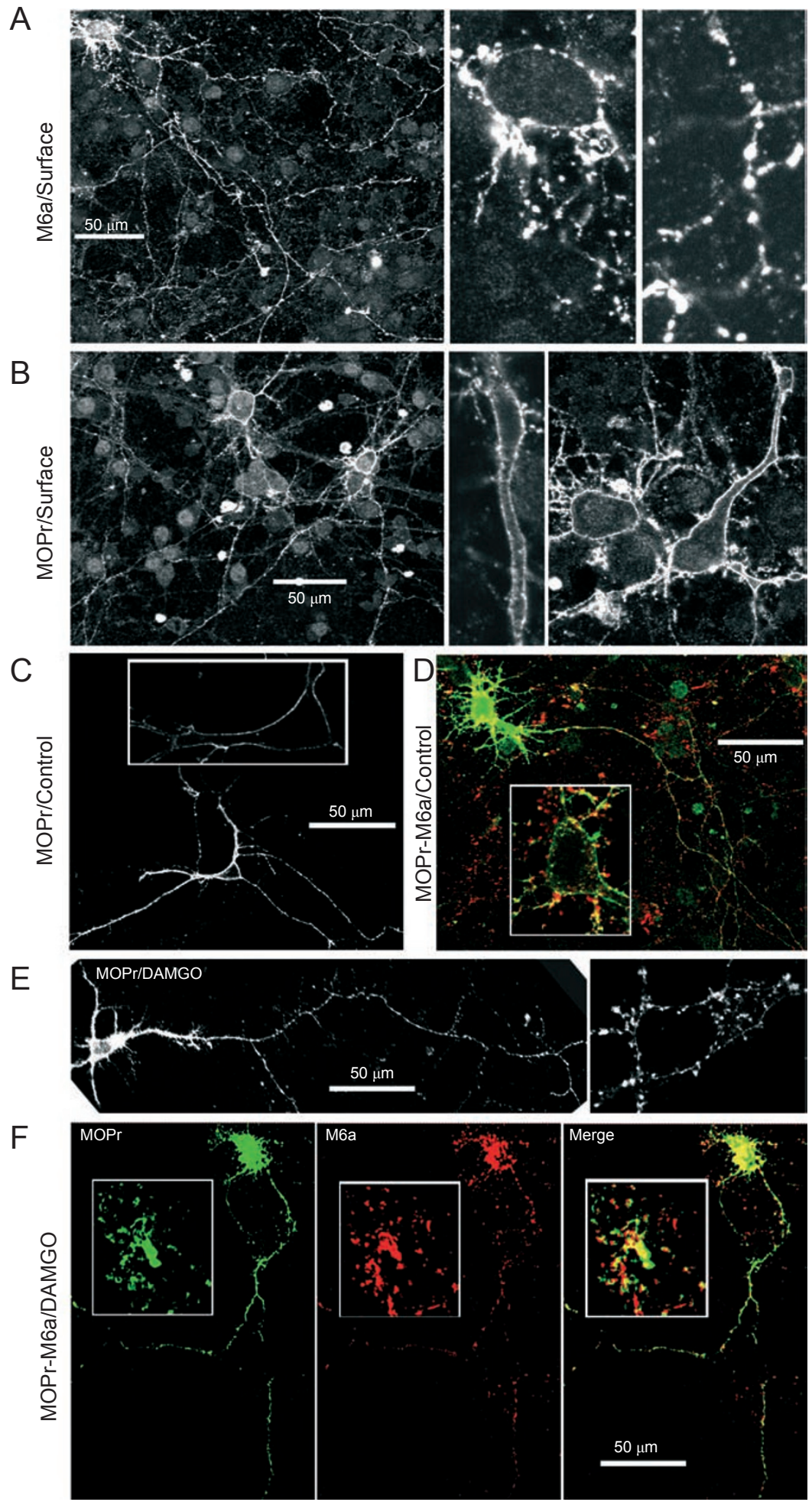

Figure 6 Co-localization and co-internalization of M6a and MOPr in cortical neurons. Newly prepared cortical neurons (E17) were transfected as described in Figure 5 and then seeded in a poly-L-lysine-coated plate. At 6 days after transfection, the cells were surface-labeled at $4{ }^{\circ} \mathrm{C}$ with anti-HA and/or ani-M6a antibody as indicated. Subsequently, the cells were left untreated (A and $\mathbf{B}$ ), treated without ( $C$ and D) or with (E and F) $10 \mu \mathrm{M}$ DAMGO in serum-free medium at $37^{\circ} \mathrm{C}$ for 30 min. Afterwards, the cells were fixed and permeabilized, and the labeled receptor and/or M6a were detected as described in 'Materials and Methods'. Co-internalization and co-localization are shown as yellow in the merged image. The cell body and/or process are shown in a higher magnification. Shown is a representative result from one of three independent experiments. 
A

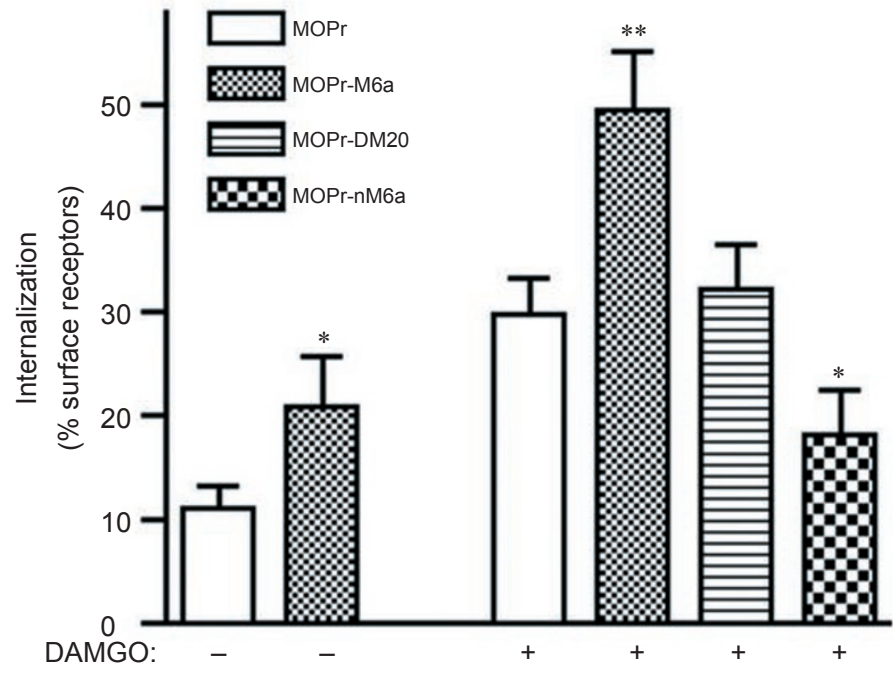

B
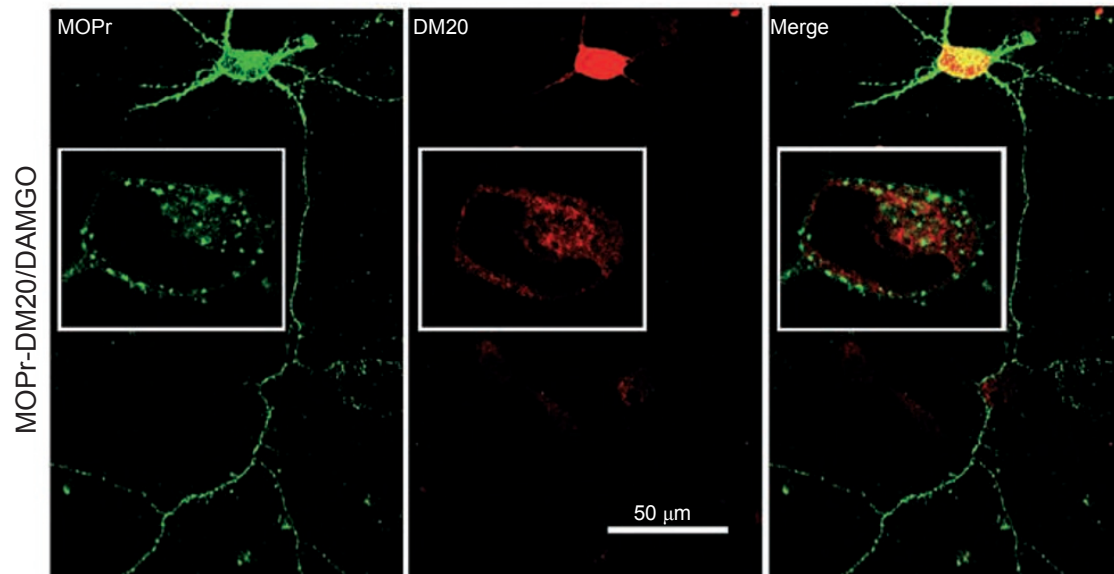

C
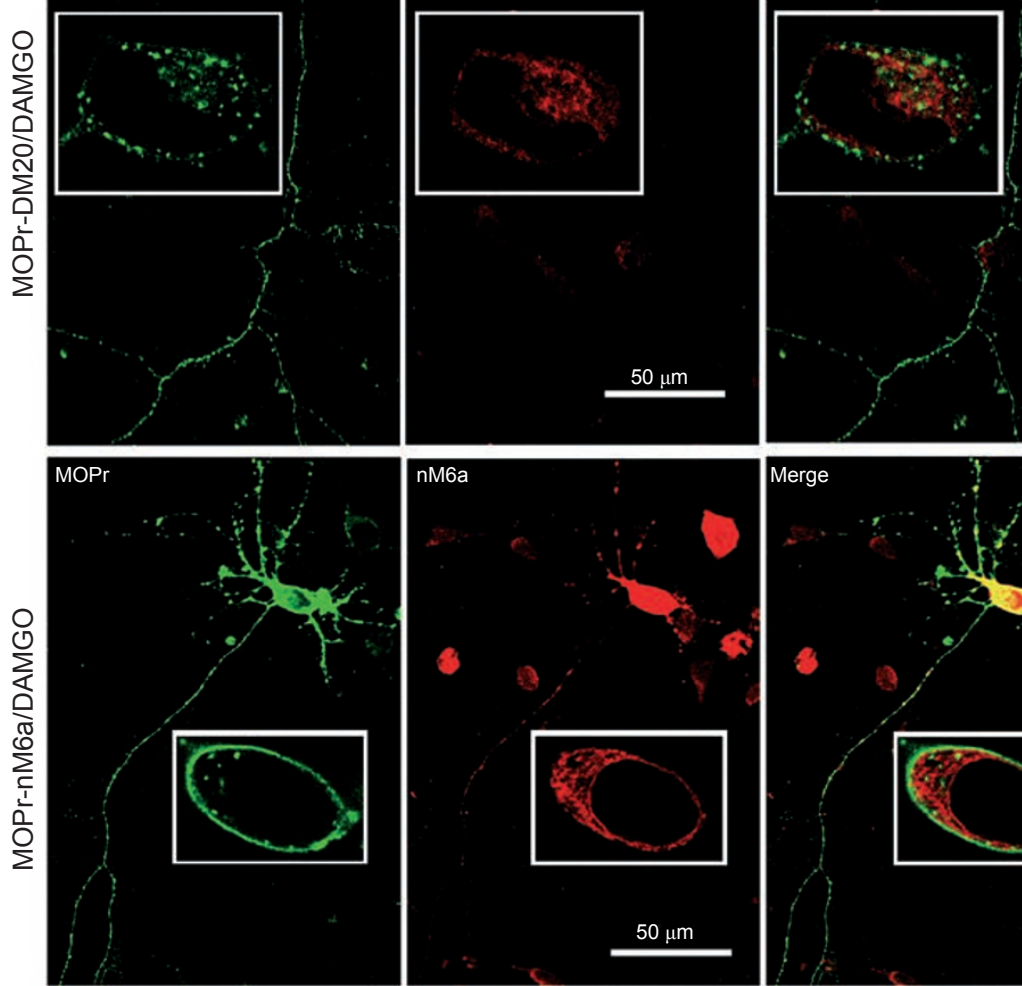

$50 \mu \mathrm{m}$
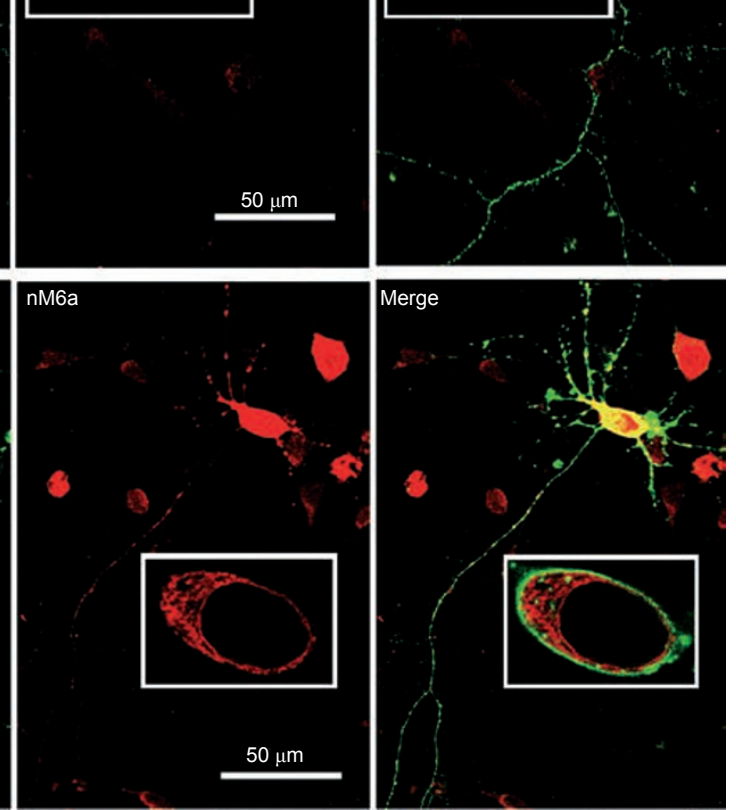

Figure 7 Co-expression of M6a augments $\mu$-opioid receptor endocytosis in cortical neurons. Newly prepared cortical neurons (E17) were co-transfected with the mixed plasmids of pEAK10-HA-MOPr plus pCMV-Myc, pCMV-Myc-M6a, pCMV-Myc-DM20 or pCMV-Myc-nM6a as indicated and then seeded in a poly-L-lysine-coated plate. (A) At 6 days after transfection, the cells were surface-labeled with anti-HA antibody. Subsequently, the cells were treated with or without $1 \mu$ M DAMGO in serum-free medium at $37^{\circ} \mathrm{C}$ for $30 \mathrm{~min}$ and then fixed. Receptor internalization, quantified as the percentage loss of cell surface receptors, was measured by ELISA. Data are presented as means \pm SEM of three independent experiments performed in triplicate. $* P<0.05$, $* * P<0.01$ versus MOPr alone, respectively (one-way ANOVA followed by Bonferroni test). (B and C) At 6 days after transfection, the cells were surface-labeled with an anti-HA antibody. Subsequently, the cells were treated with $10 \mu \mathrm{M}$ DAMGO in serum-free medium at $37^{\circ} \mathrm{C}$ for $30 \mathrm{~min}$. Afterwards, the cells were fixed, permeabilized and stained with an anti-Myc antibody. The MOPr, DM20 and/or nM6a were detected as described in 'Materials and Methods'. The cell body is shown with a higher magnification. Shown is a representative result from one of three independent experiments. 
present findings, together with our previous work [17], indicate that M6a could function as a scaffolding, anchoring, or adaptor protein in the intracellular trafficking of certain GPCRs and thus demonstrate a new function for a protein in the PLP family.

In transfected HEK293 cells, hypertonic sucrose, an inhibitor of receptor internalization [31], significantly increased M6a distribution at the plasma membrane, whereas monensin, an inhibitor of vesicle recycling [32, 33], greatly decreased the surface localization and increased the intracellular distribution of M6a. These findings suggest that M6a is a constitutive trafficking molecule that may affect the constitutive trafficking of its interacting proteins. Indeed, both in HEK293 cells and in primary cortical neurons, co-expression of M6a augmented the intracellular distribution of MOPrs.

After internalization, the regulation of opioid receptor sorting between recycling and degradation pathways is of particular interest owing to its importance in cell signaling in response to opioids. MOPr and DOPr differ significantly in their post-endocytotic sorting. Internalized DOPr is mainly targeted to lysosomes for degradation, whereas MOPr and its splice variants have distinct properties that direct them to recycle back to the cell surface upon agonist treatment $[18-20,34]$. As yet, the mechanism by which post-endocytotic sorting of opioid receptors occurs is still obscure.

A protein termed GASP (GPCR-associated sorting protein), which binds preferentially to the carboxyl tail of the DOPr, was identified as a candidate for a post-endocytotic sorting protein [16]. The interaction of DOPr with GASP directs the internalized receptors into the lysosomal pathway destined for proteolytic degradation. Our data suggest that the interaction of MOPr or DOPr with M6a facilitates the sorting of internalized receptors into the recycling pathway for reactivation and impairs the sorting of receptors into lysosomes for degradation. It is reasonable to propose that the differential post-endocytotic sorting of MOPr and DOPr could be caused by selective binding of M6a and GASP to the receptors. In support of this idea, GASP binds much less strongly to MOPr than to DOPr [16], and we observed a lower interaction of M6a with DOPr compared to MOPr [17]. We speculate that interaction of MOPr with M6a induces recycling of MOPr, whereas the interaction of DOPr with GASP targets DOPr to lysosomes for degradation. This hypothesis is further supported by the finding that over-expression of M6a leads to the sorting of internalized DOPr into the recycling pathway (Figure $3 \mathrm{~B}$ and $3 \mathrm{C}$ ).

The detailed molecular mechanism of M6a-mediated receptor trafficking is still under investigation. It is possible that M6a influences the formation of the actin cytoskeletal network and thus induces receptor transport into the cytoplasm. In support of this hypothesis, M6a was observed to be selectively enriched in the actin-rich lamellipodia and philopodia at the leading edge of neuronal growth cones [35] and transported to these sites in an actin-dependent manner [36]. Additionally, it has been suggested that an association with the actin cytoskeleton is a common property of members of the PLP family [37]. It is also possible that M6a might change some property of clathrin-coated vesicles and endosomes, for example charge or $\mathrm{pH}$, and thereby influence vesicle transport and endosome sorting. An acidified endosome is important for the recycling of internalized receptors $[32,33]$. In support of this hypothesis, tetraspan proteolipids, including members of the PLP family, are known to contribute to the formation of pore structures in a wide spectrum of cellular systems [38]. Moreover, Rhombex-29, a new member of the PLP family, was suggested to be responsible for transporting $\mathrm{H}^{+}$[39].

In conclusion, we have demonstrated that M6a functions in opioid receptor trafficking both in HEK293 cells and in primary cultured neurons. M6a might also modulate the intracellular trafficking of certain other receptors since M6a interacts with a number of GPCRs [17]. It has been well documented that MOPr endocytosis reduces the development of opioid tolerance by promoting fast receptor resensitization and recycling [12, 40-47]. It is therefore reasonable to suggest that M6a-enhanced endocytosis/recycling of MOPrs attenuates the development of opiate tolerance.

\section{Materials and Methods}

\section{Epitope tagging and cloning of cDNA}

The MOPr (Gi: 6981309) and $\delta$-opioid receptor (Gi: 31543370$)$ were tagged at the amino terminus with the influenza hemagglutinin-HA epitope tag (YPYDVPDYA) using polymerase chain reaction (PCR); these fragments were then subcloned into the pEAK10 expression vector containing the puromycin resistance gene (Edge Bio Systems, Gaithersburg, MD, USA) to generate the plasmids pEAK10-HA-MOPr and pEAK10-HA-DOPr, respectively. As described previously [17], the coding region of M6a (GI: 31981997), M6a negative truncation mutant (nM6a, amino acid 129-255 of M6a) or DM20 (GI: 200408) was subcloned into pCMV-Myc (Clontech, Heidelberg, Germany) by PCR to generate an amino-terminally Myctagged M6a, nM6a or DM20. The Myc-M6a was further subcloned into the pcDNA3.1 expression vector containing the hygromycin resistance gene (Invitrogen, Karlsruhe, Germany) by PCR.

\section{Cell culture}

HEK293 cells were maintained in DMEM (BF12-604F, Cambrex, Verviers, Belgium) supplemented with $10 \%$ fetal bovine serum (FBS) (S0115, Biochrom AG, Berlin, Germany) and relevant antibiotics in a humidified incubator with an atmosphere containing $10 \% \mathrm{CO}_{2}$ at $37{ }^{\circ} \mathrm{C}$. For all experiments, cells were grown overnight in DMEM supplemented with $10 \%$ FBS without any antibiotics and pre-incu- 
bated in UltraMEM (BE12-743F, Cambrex) for about 30 min prior to different treatments. Unless otherwise specified, all cells were treated in UltraMEM.

\section{Generation of stable cell lines}

According to the manufacturer's protocol, HEK293 cells were first transfected with pEAK10-HA-MOPr, pEAK10-HA-DOPr or pcDNA3.1-Myc-M6a plasmid using Lipofectamine ${ }^{\mathrm{TM}} 2000$ (Invitrogen, Karlsruhe, Germany). Stable transfectants were selected in the presence of $1.25 \mu \mathrm{g} / \mathrm{ml}$ puromycin (Sigma, Deisenhofen, Germany) or $400 \mu \mathrm{g} / \mathrm{ml}$ hygromycin B (PAA Laboratories $\mathrm{GmbH}$, Pasching, Germany). To generate a stable cell line co-expressing HA-MOPr and Myc-M6a, stable HA-MOPr cells were subjected to a second round of transfection with pcDNA3.1-Myc-M6a plasmid as described above and selected in the presence of $1.25 \mu \mathrm{g} / \mathrm{ml}$ puromycin and $400 \mu \mathrm{g} / \mathrm{ml}$ hygromycin B. Stable lines expressing similar densities of both receptors were used for further study. Receptor and/or M6a expression were monitored using confocal microscopy and western blot analysis.

\section{Immunocytochemistry}

HEK293 cells were grown overnight on poly-L-lysine-treated coverslips. After treatment or without treatment, the cells were fixed with Zamboni's fixative (4\% paraformaldehyde and $0.2 \%$ picric acid in phosphate buffer, $\mathrm{pH}$ 6.9) for $20 \mathrm{~min}$ at room temperature (RT). After a wash with TPBS (10 mM Tris, $10 \mathrm{mM}$ phosphate buffer, 137 $\mathrm{mM} \mathrm{NaCl}$ and $0.05 \%$ thimerosal, $\mathrm{pH} 7.4$ ), specimens were incubated for $3 \mathrm{~min}$ each in $50 \%$ and $100 \%$ methanol to permeabilize the membrane. Subsequently, specimens were washed several times in TPBS and then pre-incubated with TPBS containing 3\% normal goat serum (NGS) at RT for $1 \mathrm{~h}$. Then, cells were incubated with mouse anti-Myc antibody (Clontech) at a concentration of $0.2 \mu \mathrm{g} / \mathrm{ml}$ in TPBS containing 1\% NGS at RT for $1 \mathrm{~h}$. Bound primary antibodies were detected with cyanine 3.18-conjugated secondary antibodies (1:400 or $3.75 \mu \mathrm{g} / \mathrm{ml}$, Jackson ImmunoResearch, West Grove, PA). Cells were then permanently mounted in DPX (Fluka, NeuUlm, Germany). Specimens were examined using a Leica TCS-NT laser-scanning confocal microscope equipped with a krypton/argon laser.

For further characterization of the endocytic pathway in $\mathrm{MOPr}$ and M6a in transfected HEK293 cells, the receptor and M6a were surface-labeled with anti-HA (Gramsch Laboratories, Schwabhausen, Germany) and anti-M6a antibodies (Medical \& Biological Laboratories, Göttingen, Germany) at a final concentration of 1.0 $\mu \mathrm{g} / \mathrm{ml}$ in UltraMEM medium at $4{ }^{\circ} \mathrm{C}$ for $1 \mathrm{~h}$. After treatment as indicated, the cells were fixed, permeabilized, and blocked as described above. Afterwards, the cells were incubated with goat anti-EEA1 or Rab 11 antibody (Santa Cruz Biotechnology, Santa Cruz, CA) at a concentration of $4 \mu \mathrm{g} / \mathrm{ml}$ in TPBS containing $1 \%$ NGS at RT for $3 \mathrm{~h}$. Antibody-labeled receptor, M6a and endogenous EEA1 (or Rab 11) were detected with a mixture of Alexa Fluor 488- (1:1,200, Molecular Probes, Invitrogen), Cyanine 3.18- (1:400, Jackson ImmunoResearch) and Alexa Fluor 647- (1:500, Molecular Probes) conjugated second antibodies, respectively. Alexa Fluor 488 was imaged with $488 \mathrm{~nm}$ excitation and $530 \mathrm{~nm}$ emission filters; Cyanine 3.18 was imaged with $568 \mathrm{~nm}$ excitation and 570-630 $\mathrm{nm}$ band pass emission filters; and Alexa Fluor 647 (or Cyanine 5.18) was imaged with $647 \mathrm{~nm}$ excitation and $665 \mathrm{~nm}$ long pass emission filters. To characterize the effect of M6a on receptor distribution in EEA1- or Rab 11-labeled vesicles, vesicles with co-localization of the receptor,
EEA1, Rab 11 and/or M6a were counted. The percentage of receptors targeted to EEA1- or Rab 11-positive endosomes in the presence or absence of M6a is shown.

Similarly, for immunocytochemistry in primary cortical neurons, the cells were fixed, and the distribution of MOPr and M6a was determined using rabbit anti-HA $(0.2 \mu \mathrm{g} / \mathrm{ml})$ and/or mouse anti-Myc $(0.2 \mu \mathrm{g} / \mathrm{ml})$ antibodies followed by Alexa Fluor 488 - and/or Cyanine 3.18-conjugated secondary antibodies. To observe the endocytosis of surface MOPr and/or M6a, cells were first surface-labeled with a rabbit anti-HA and/or a rat anti-M6a antibody at a final concentration of $2.0 \mu \mathrm{g} / \mathrm{ml}$ in UltraMEM medium at $4{ }^{\circ} \mathrm{C}$ for $30 \mathrm{~min}$ at 6 days posttransfection. Subsequently, the cells were incubated in the presence or

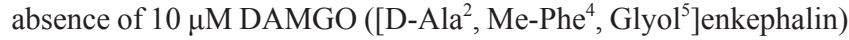
for $30 \mathrm{~min}$ prior to fixation and permeabilization. The bound primary antibodies were detected with Alexa Fluor 488- and/or Cyanine 3.18conjugated secondary antibodies as described above.

\section{Transferrin trafficking}

A previously described 'pulse-chase' assay [34, 48] was used to estimate the degree to which a 'pulse' of internalized MOPr/DOPr and M6a was accessible to a subsequent 'chase' of endocytosed transferrin. In short, receptors and M6a were surface-labeled with anti-HA and anti-M6a antibodies $(1 \mu \mathrm{g} / \mathrm{ml}$, each $)$ at $4{ }^{\circ} \mathrm{C}$ for $1 \mathrm{~h}$. Cells were first incubated with agonist at $37^{\circ} \mathrm{C}$ for $30 \mathrm{~min}$ to drive internalization of antibody-labeled receptor and M6a and then rinsed with EDTAsupplemented phosphate-buffered saline. Subsequently, cells were rewarmed to $37{ }^{\circ} \mathrm{C}$ for $20 \mathrm{~min}$ in agonist-free medium containing $5 \mu \mathrm{g} / \mathrm{ml}$ Alexa Fluor 488-conjugated transferrin (Molecular Probes, Eugene, Oregon, USA). Finally, cells were fixed and permeabilized, and antibody-labeled receptor and M6a were detected with a mixture of Cyanine 5.18- and Cyanine 3.18-conjugated second antibodies, as described above.

\section{Radioligand binding assays}

The binding studies were performed by saturation binding assays on total proteins prepared from stably transfected HEK293 cells expressing HA-MOPr with and without co-expression of Myc-M6a. The number of $\left[{ }^{3} \mathrm{H}\right] \mathrm{DAMGO}$ binding sites $\left(B_{\max }\right)$ was calculated by Scatchard analysis using at least seven concentrations of $\left[{ }^{3} \mathrm{H}\right] \mathrm{DAMGO}$ in a range from 0.25 to $10 \mathrm{nM}$ as previously described [49]. Nonspecific binding was determined as radioactivity bound in the presence of $1 \mu \mathrm{M}$ unlabeled DAMGO. The radioligand binding assay was sensitive enough to calculate the receptor amount. The number of $\left[{ }^{3} \mathrm{H}\right] \mathrm{DAMGO}$ binding sites in a membrane binding assay reflects the amount of total MOPrs, which can be used to evaluate the receptor down-regulation.

\section{Primary cultures of the cerebral cortex and transfection}

All animal procedures were approved by the Otto-von-Guericke-University Magdeburg. Cortical cultures were obtained from E17 Sprague Dawley rat embryos (Charles River) and prepared as described previously [50]. The freshly prepared cortical cells were transfected with pEAK10-HA-MOPr, pCMV-Myc-M6a, pCMVMyc-nM6a and/or pCMV-Myc-DM20 plasmids using Rat Neuron Nucleofector Solution according to the manufacturer's protocol (Amaxa Inc., USA). After transfection, the cells were seeded at about $6 \times 10^{5}$ cells per well in a 12 -well plate with poly-L-lysine-coated coverslips for immunocytochemistry and at about $4 \times 10^{5}$ cells per well in a 24-well poly-L-lysine-coated plate for quantitative assays. The 
cells grew in Neurobasal medium (Invitrogen, Paisley, UK) supplemented with $2 \%$ B-27, $0.5 \mathrm{mM} \mathrm{L}$-glutamine, $100 \mathrm{U} / \mathrm{ml}$ penicillin and $100 \mu \mathrm{g} / \mathrm{ml}$ streptomycin. Cells were cultured at $37{ }^{\circ} \mathrm{C}$ and $5 \% \mathrm{CO}_{2}$ in a humidified incubator. On the fourth day after seeding the cells and then every second day thereafter, $50 \%$ of the culture medium was replaced. Cells were used for experiments 6 days after transfection. Neurons, astrocytes and oligodendrocytes in the cultures were characterized by immunocytochemistry using antibodies for MAP2 (M4403, Sigma), GFAP (GP52, Progen, Heidelberg, Germany) and CNPase (C5922, Sigma), respectively.

\section{Quantitative analysis of receptor internalization}

Newly prepared cortical cells (E17) were co-transfected with the mixed plasmids of pEAK10-HA-MOPr plus pCMV-Myc, pCMVMyc-M6a, pCMV-Myc-DM20 or pCMV-Myc-nM6a as indicated, and then seeded at $4 \times 10^{5}$ cells per well in a poly-L-lysine-coated 24-well plate. At 6 days after transfection, the cells were precultured in UltraMEM medium for 30 min and then surface-labeled with an anti-HA antibody as described above. Subsequently, the cells were treated with or without $1 \mu \mathrm{M}$ DAMGO in UltraMEM medium at $37^{\circ} \mathrm{C}$ for $30 \mathrm{~min}$ and then fixed. Following fixation and wash, the cells were incubated with peroxidase-conjugated anti-rabbit antibody $(1: 1,000$, Amersham) in UltraMEM for $2 \mathrm{~h}$ at RT. Plates were developed with $250 \mu$ of ABTS solution (Roche Molecular Biomedicals, Mannheim, Germany). After $15 \mathrm{~min}, 200 \mu \mathrm{l}$ of the substrate solution from each well was transferred to a 96-well plate and analyzed at $405 \mathrm{~nm}$ using an Expert Plus Microplate Reader (ASYS, Eugendorf, Austria). Receptor endocytosis was evaluated as the loss of surface-labeled receptors.

\section{Acknowledgments}

We thank Karina Schäfer, Michaela Böx and Evelyn Kahl (Department of Pharmacology and Toxicology, Otto-von-Guericke-University, Magdeburg, Germany) for excellent technical assistance. This work was supported by grants HO 1027/10-1 and SFB 426/A2 from the Deutsche Forschungsgemeinschaft (to $\mathrm{VH}$ ) and BMBFgrant 01ZZ0407 (to TK).

\section{References}

1 von Zastrow M. A cell biologist's perspective on physiological adaptation to opiate drugs. Neuropharmacology 2004; 47 Suppl 1:286-292.

2 Kieffer BL. Recent advances in molecular recognition and signal transduction of active peptides: receptors for opioid peptides. Cell Mol Neurobiol 1995; 15:615-635.

3 Matthes HW, Maldonado R, Simonin F, et al. Loss of morphineinduced analgesia, reward effect and withdrawal symptoms in mice lacking the mu-opioid-receptor gene. Nature 1996; 383:819823.

4 Kieffer BL. Opioids: first lessons from knockout mice. Trends Pharmacol Sci 1999; 20:19-26.

5 Kieffer BL, Gaveriaux-Ruff C. Exploring the opioid system by gene knockout. Prog Neurobiol 2002; 66:285-306.

6 von Zastrow M, Svingos A, Haberstock-Debic H, Evans C. Regulated endocytosis of opioid receptors: cellular mechanisms and proposed roles in physiological adaptation to opiate drugs. Curr Opin Neurobiol 2003; 13:348-353.

7 Ferguson SS. Evolving concepts in G protein-coupled receptor endocytosis: the role in receptor desensitization and signaling. Pharmacol Rev 2001; 53:1-24.

8 Claing A, Laporte SA, Caron MG, Lefkowitz RJ. Endocytosis of G protein-coupled receptors: roles of G protein-coupled receptor kinases and beta-arrestin proteins. Prog Neurobiol 2002; 66:6179.

9 Mousavi SA, Malerod L, Berg T, Kjeken R. Clathrin-dependent endocytosis. Biochem J 2004; 377:1-16.

10 Lefkowitz RJ, Shenoy SK. Transduction of receptor signals by beta-arrestins. Science 2005; 308:512-517.

11 Shenoy SK, Lefkowitz RJ. Seven-transmembrane receptor signaling through beta-arrestin. Sci STKE 2005; 2005:cm10.

12 Liang YJ, Wu DF, Yang LQ, Hollt V, Koch T. Interaction of the mu-opioid receptor with synaptophysin influences receptor trafficking and signaling. Mol Pharmacol 2007; 71:123-131.

13 Heydorn A, Sondergaard BP, Ersboll B, et al. A library of 7TM receptor C-terminal tails. Interactions with the proposed postendocytic sorting proteins ERM-binding phosphoprotein 50 (EBP50), N-ethylmaleimide-sensitive factor (NSF), sorting nexin 1 (SNX1), and G protein-coupled receptor-associated sorting protein (GASP). J Biol Chem 2004; 279:54291-54303.

14 Onoprishvili I, Andria ML, Kramer HK, et al. Interaction between the mu opioid receptor and filamin A is involved in receptor regulation and trafficking. Mol Pharmacol 2003; 64:1092-1100.

15 Koch T, Brandenburg LO, Schulz S, et al. ADP-ribosylation factor-dependent phospholipase D2 activation is required for agonist-induced mu-opioid receptor endocytosis. $J$ Biol Chem 2003; 278:9979-9985.

16 Whistler JL, Enquist J, Marley A, et al. Modulation of postendocytic sorting of G protein-coupled receptors. Science 2002; 297:615-620.

$17 \mathrm{Wu}$ DF, Koch T, Liang YJ, et al. Membrane glycoprotein M6a interacts with the mu-opioid receptor and facilitates receptor endocytosis and recycling. J Biol Chem 2007; 282:22239-22247.

18 Wang W, Loh HH, Law PY. The intracellular trafficking of opioid receptors directed by carboxyl tail and a di-leucine motif in Neuro2A cells. J Biol Chem 2003; 278:36848-36858.

19 Marie N, Lecoq I, Jauzac P, Allouche S. Differential sorting of human delta-opioid receptors after internalization by peptide and alkaloid agonists. J Biol Chem 2003; 278:22795-22804.

20 Tanowitz M, von Zastrow M. A novel endocytic recycling signal that distinguishes the membrane trafficking of naturally occurring opioid receptors. J Biol Chem 2003; 278:45978-45986.

21 Dunn KW, McGraw TE, Maxfield FR. Iterative fractionation of recycling receptors from lysosomally destined ligands in an early sorting endosome. J Cell Biol 1989; 109:3303-3314.

22 Hopkins CR, Trowbridge IS. Internalization and processing of transferrin and the transferrin receptor in human carcinoma A431 cells. J Cell Biol 1983; 97:508-521.

23 Werner H, Dimou L, Klugmann M, Pfeiffer S, Nave KA. Multiple splice isoforms of proteolipid M6B in neurons and oligodendrocytes. Mol Cell Neurosci 2001; 18:593-605.

24 Keith DE, Anton B, Murray SR, et al. mu-Opioid receptor internalization: opiate drugs have differential effects on a conserved endocytic mechanism in vitro and in the mammalian brain. Mol Pharmacol 1998; 53:377-384. 
25 Yan Y, Lagenaur C, Narayanan V. Molecular cloning of M6: identification of a PLP/DM20 gene family. Neuron 1993; 11:423431.

26 Yan Y, Narayanan V, Lagenaur C. Expression of members of the proteolipid protein gene family in the developing murine central nervous system. J Comp Neurol 1996; 370:465-478.

27 Roussel G, Trifilieff E, Lagenaur C, Nussbaum JL. Immunoelectron microscopic localization of the M6a antigen in rat brain. $J$ Neurocytol 1998; 27:695-703.

28 Lagenaur C, Kunemund V, Fischer G, Fushiki S, Schachner M. Monoclonal M6 antibody interferes with neurite extension of cultured neurons. J Neurobiol 1992; 23:71-88.

29 Alfonso J, Fernandez ME, Cooper B, Flugge G, Frasch AC. The stress-regulated protein M6a is a key modulator for neurite outgrowth and filopodium/spine formation. Proc Natl Acad Sci USA 2005; 102:17196-17201.

30 Mukobata S, Hibino T, Sugiyama A, et al. M6a acts as a nerve growth factor-gated $\mathrm{Ca}(2+)$ channel in neuronal differentiation. Biochem Biophys Res Commun 2002; 297:722-728.

31 Heuser JE, Anderson RG. Hypertonic media inhibit receptormediated endocytosis by blocking clathrin-coated pit formation. $J$ Cell Biol 1989; 108:389-400.

32 Stein BS, Bensch KG, Sussman HH. Complete inhibition of transferrin recycling by monensin in K562 cells. J Biol Chem 1984; 259:14762-14772.

33 Kaiser J, Stockert RJ, Wolkoff AW. Effect of monensin on receptor recycling during continuous endocytosis of asialoorosomucoid. Exp Cell Res 1988; 174:472-480.

34 Tsao PI, von Zastrow M. Type-specific sorting of G protein-coupled receptors after endocytosis. J Biol Chem 2000; 275:1113011140.

35 Baumrind NL, Parkinson D, Wayne DB, Heuser JE, Pearlman AL. EMA: a developmentally regulated cell-surface glycoprotein of CNS neurons that is concentrated at the leading edge of growth cones. Dev Dyn 1992; 194:311-325.

36 Sheetz MP, Baumrind NL, Wayne DB, Pearlman AL. Concentration of membrane antigens by forward transport and trapping in neuronal growth cones. Cell 1990; 61:231-241.

37 Kalwy SA, Smith R, Kidd GJ. Myelin proteolipid protein expressed in COS-1 cells is targeted to actin-associated surfaces. J Neurosci Res 1997; 48:201-211.
38 Kitagawa K, Sinoway MP, Yang C, Gould RM, Colman DR. A proteolipid protein gene family: expression in sharks and rays and possible evolution from an ancestral gene encoding a poreforming polypeptide. Neuron 1993; 11:433-448.

39 Shimokawa N, Miura M. Rhombex-29, a novel gene of the PLP/DM20-M6 family cloned from rat medulla oblongata by differential display. J Neurosci Res 2000; 62:1-8.

40 Koch T, Brandenburg LO, Liang Y, et al. Phospholipase D2 modulates agonist-induced mu-opioid receptor desensitization and resensitization. $J$ Neurochem 2004; 88:680-688.

41 Finn AK, Whistler JL. Endocytosis of the mu opioid receptor reduces tolerance and a cellular hallmark of opiate withdrawal. Neuron 2001; 32:829-839.

42 He L, Fong J, von Zastrow M, Whistler JL. Regulation of opioid receptor trafficking and morphine tolerance by receptor oligomerization. Cell 2002; 108:271-282.

43 Whistler JL, Chuang HH, Chu P, Jan LY, von Zastrow M. Functional dissociation of mu opioid receptor signaling and endocytosis: implications for the biology of opiate tolerance and addiction. Neuron 1999; 23:737-746.

44 Koch T, Widera A, Bartzsch K, et al. Receptor endocytosis counteracts the development of opioid tolerance. Mol Pharmacol 2005; 67:280-287.

45 Lefkowitz RJ, Pitcher J, Krueger K, Daaka Y. Mechanisms of beta-adrenergic receptor desensitization and resensitization. $A d v$ Pharmacol 1998; 42:416-420.

46 Law PY, Erickson LJ, El-Kouhen R, et al. Receptor density and recycling affect the rate of agonist-induced desensitization of mu-opioid receptor. Mol Pharmacol 2000; 58:388-398.

47 Ferguson SS, Zhang J, Barak LS, Caron MG. Molecular mechanisms of $\mathrm{G}$ protein-coupled receptor desensitization and resensitization. Life Sci 1998; 62:1561-1565.

48 Tulipano G, Stumm R, Pfeiffer M, et al. Differential beta-arrestin trafficking and endosomal sorting of somatostatin receptor subtypes. J Biol Chem 2004; 279:21374-21382.

49 Koch T, Wu DF, Yang LQ, Brandenburg LO, Hollt V. Role of phospholipase D2 in the agonist-induced and constitutive endocytosis of G-protein coupled receptors. J Neurochem 2006; 97:365-372.

$50 \mathrm{Wu}$ DF, Yang LQ, Goschke A, et al. Role of receptor internalization in the agonist-induced desensitization of cannabinoid type 